\title{
Relation entre la mastication et la qualité de l'alimentation des aînés de l'Étude longitudinale québécoise sur la nutrition et le vieillissement (NuAge)*
}

\author{
Valérie Cusson, ${ }^{1}$ Hélène Payette, ${ }^{2}$ Christian Caron, ${ }^{3}$ Bryna Shatenstein, ${ }^{4}$ and Pierrette Gaudreau ${ }^{5}$
}

\begin{abstract}
The objective of this study was to assess the relationship between the quality of mastication and dietary intake among community-dwelling older adults (67-84 years). A secondary analysis of data collected upon entry of participants in the NuAge study was effected. Perceived masticatory efficiency $(n=1793)$ was measured using a questionnaire inspired by the Oral Health Impact Profile. Direct assessment of masticatory efficiency was carried out in a subsample (n=94), using a validated clinical test (Swallowing Threshold Test Index, 0-100\%). Dietary intake was calculated from three non-consecutive $24 \mathrm{~h}$ recalls. Mean scores indicate good perceived masticatory efficiency (Men:26.22 $\pm 0.19 / 28 ;$ Women:25.81 $\pm 0.21 / 28$ ), but weak assessed efficiency (Men:60.8\% [CI(57.3-64.2)]; Women:61.2\% [CI(57.7-64.7)]). Multivariate regression analysis, adjusting for confounding variables (age, sex, schooling, living alone, income, smoking, chronic conditions, functional autonomy), showed that masticatory efficiency (measured or perceived) was not associated with intake of fruits/ vegetables, protein, vitamin $\mathrm{C}$ or folic acid.
\end{abstract}

\section{RÉSUMÉ}

L'objectif de cette étude était de vérifier les relations entre la qualité de la mastication et les apports alimentaires chez des aînés (67-84 ans) vivant dans la communauté. Une analyse secondaire des données recueillies à l'entrée des participants dans l'étude NuAge a été réalisée. La perception de l'efficacité masticatoire ( $\mathrm{n}=1793)$ a été mesurée à l'aide d'un questionnaire inspiré du Oral Health Impact Profile et l'efficacité masticatoire à l'aide d'un test clinique (Swallowing Threshold Test Index, 0-100\%) dans un sous-échantillon ( $\mathrm{n}=94)$. Les apports alimentaires ont été calculés à partir de 3 rappels de $24 \mathrm{~h}$ non-consécutifs. En moyenne, malgré une bonne perception de l'efficacité masticatoire (Hommes:26,22 $\pm 0,19 / 28$;Femmes:25,81 $\pm 0,21 / 28$ ), les résultats indiquent une faible efficacité masticatoire (Hommes: 60,8\%[IC(57,3-64,2)];Femmes:61,2\%[IC(57,7-64,7)]. Des analyses de régression multivariées, ajustées pour les variables de confusion (âge, sexe, scolarité, vivre seul, revenu, tabagisme, conditions chroniques, autonomie fonctionnelle), montrent que l'efficacité masticatoire (mesurée ou perçue) n'était pas associée aux apports en fruits/légumes, protéines, vitamine $\mathrm{C}$ et en folate.

1 Centre de recherche sur le vieillissement, CSSS-IUGS, Université de Sherbrooke, Sherbrooke (Québec, Canada)

2 Centre de recherche sur le vieillissement, CSSS-IUGS, Département de santé communautaire, Faculté de médecine et des sciences de la santé, Université de Sherbrooke, Sherbrooke (Québec, Canada)

3 Centre d'excellence sur le vieillissement de Québec (CEVQ) du CHU de Québec, Faculté de médecine dentaire, Université Laval, Québec (Québec, Canada)

4 Centre de recherche de l'Institut universitaire de gériatrie de Montréal, Département de nutrition, Université de Montréal, Montréal (Québec, Canada)

5 Centre de recherche du Centre Hospitalier de l’Université de Montréal, Faculté de médecine, Université de Montréal, Montréal (Québec, Canada)

* Valérie Cusson a reçu un soutien financier du Réseau de recherche en santé buccodentaire et osseuse (RSBO), un Réseau thématique du Fonds de recherche du Québec-Santé et de la chaire de recherche Merck-Frosst en pharmaco-gériatrie

Manuscript received: / manuscrit reçu : 11/09/14

Manuscript accepted: / manuscrit accepté : 28/01/15

Mots-clés : alimentation, mastication, aînés

Keywords: food intake, masticatory efficiency, older adults 
La correspondance et les demandes de tirés-à-part doivent être adressées à: / Correspondence and requests for offprints should be sent to:

Hélène Payette, Ph.D.

Centre de recherche sur le vieillissement, CSSS-IUGS

1036, rue Belvédère Sud

Sherbrooke, QC J1H 4C4

(helene.payette@usherbrooke.ca)

\section{Introduction}

Le vieillissement a plusieurs effets sur la santé générale, incluant la condition dentaire et l'état nutritionnel. Pour favoriser un vieillissement sain il importe de se préoccuper de tous ces aspects. Toutefois, ces derniers sont deux domaines souvent négligés dans la pratique clinique. Par conséquent, les problèmes dentaires et nutritionnels sont fréquents chez les aînés qui constituent un groupe à risque au plan nutritionnel (Keller, Østbye, \& Goy, 2004). On rapporte que 34\% des Canadiens de 65 ans et plus présentent un risque nutritionnel, selon les résultats de l'Enquête sur la santé dans les collectivités canadiennes - Vieillissement en santé, menée en 2008-2009 (Ramage-Morin \& Garriguet, 2013; Statistique Canada, 2011). De plus, les personnes âgées consultent peu les dentistes pour des raisons économiques, à cause d'une perception erronée de leurs problèmes dentaires ou en raison de la difficulté d'accès aux services de soins dentaires (Centre d'excellence pour la santé buccodentaire et le vieillissement, 2006; Ordre des dentistes du Québec, 2007). Pourtant, elles présentent plusieurs problèmes de santé buccodentaire comme l'édentation, les prothèses dentaires inadéquates, les caries dentaires, les racines résiduelles, la xérostomie ou une piètre hygiène buccale (Arpin, Brodeur, \& Corbeil, 2008). L'importance d'une bonne alimentation (Santé Canada, 2010) et d'une bonne santé buccodentaire (Gonsalves, Wrightson, \& Henry, 2008; Rose, Steinberg, \& Minsk, 2000; Santé Canada, 2009; Shay, 2002; Taylor, Loesche, \& Terpenning, 2000) sur l'état de santé générale est de plus en plus reconnue. Déjà en 1982, la Fédération Dentaire Internationale établissait des objectifs spécifiques quant à la santé dentaire à atteindre pour l'an 2000. L'un d'eux visait la réduction de $25 \%$ du niveau d'édentation ainsi que la rétention d'un minimum de 20 dents fonctionnelles chez au moins 50\% des personnes âgées de 65 ans et plus. Selon la Fédération Dentaire Internationale, l'atteinte de ces objectifs devait permettre de réduire la douleur et les infections buccales, en plus d'améliorer grandement la qualité de vie de la population âgée (Fédération Dentaire Internationale, 1982). Des liens directs ont été démontrés entre un état buccodentaire médiocre et des problèmes pulmonaires, cardiaques et un piètre contrôle du diabète (Gonsalves et al., 2008; Rose et al., 2000; Santé Canada, 2009; Shay, 2002; Taylor et al., 2000). De plus, l'état de santé dentaire, et plus spécifiquement la mastication, peut influencer de façon importante la consommation d'aliments et l'état nutritionnel. Le nombre de dents présentes en bouche et les problèmes masticatoires influencent les choix et la consommation d'aliments (Lee, Huang, \& Wahlquist, 2010; Sahyoun, Lin, \& Krall, 2003; Sheiham, Steele, Marcenes, Finch, \& Walls, 1999). De plus faibles apports en fruits et en viande ont été observés chez les participants ayant des difficultés masticatoires comparativement à ceux ayant une bonne efficacité masticatoire (Lee et al., 2010). De plus, plusieurs auteurs ont fait le constat qu'avoir un nombre réduit de paires dentaires occlusives (PDO), c'est-à-dire les dents postérieures antagonistes qui entrent en contact lorsqu'on ferme la bouche, était relié à une efficacité masticatoire réduite tandis que l'efficacité augmentait avec le nombre de PDO (Marcenes, Steele, Sheiham, \& Walls, 2003; Sheiham et al., 1999). La présence d'un nombre élevé de PDO permet de consommer des aliments plus difficiles à mastiquer (Sahyoun et al., 2003). En effet, les personnes ayant un plus grand nombre de PDO, comparativement à celles en ayant peu ou pas, présentent moins de difficultés à mastiquer une pomme, une carotte crue, des noix et une grillade bien cuite (Sheiham et al., 1999). Les fruits et les légumes sont généralement difficiles à mastiquer ainsi que les aliments fournissant les protéines (ex. viandes, noix), la vitamine $C$ (ex. orange crue) et le folate (ex. laitue fraîche). La perte de dents et les problèmes masticatoires entraînent également des choix de textures différentes d'aliments, faciles à mastiquer et généralement moins denses en nutriments, notamment en protéines et en fibres (Sahyoun et al., 2003), ainsi qu'en vitamine $D$, thiamine, niacine, acide pantothénique et pyridoxine $\left(\mathrm{B}_{6}\right)$ (Yoshihara, Watanabe, Nishimuta, Hanada, \& Miyazaki, 2005). La santé buccodentaire et l'efficacité masticatoire chez les aînés pourraient donc influencer significativement leur état nutritionnel (Sheiham et al., 1999). De plus en plus de recherches (N'Gom \& Woda, 2002) se font dans le domaine de la nutrition et de la santé dentaire, mais peu d'études canadiennes (Shatenstein, 1986; Brodeur, Laurin, Vallée, \& Lachapelle, 1993) ont été réalisées sur la relation entre la mastication et l'état nutritionnel, notamment chez les personnes âgées vivant dans la communauté.

L'objectif général de cette étude était donc de déterminer la relation entre l'efficacité masticatoire et les apports 
alimentaires chez des personnes âgées généralement en bonne santé. Plus spécifiquement, nous voulions examiner la relation entre l'efficacité masticatoire (mesurée et perçue) et l'apport alimentaire de protéines totales, de vitamine $C$ et de folate ainsi que la consommation de fruits et légumes.

\section{Méthodologie}

Population à l'étude

Une analyse secondaire des données recueillies à l'entrée des participants dans l'Étude longitudinale québécoise (2003-2005) sur la nutrition comme déterminant d'un vieillissement réussi (NuAge) a été réalisée. L'étude NuAge a recruté une cohorte de 1793 hommes et femmes âgés de 67 à 84 ans qui ont été suivis annuellement pendant 4 ans (Gaudreau et al., 2007). L'échantillon, stratifié pour l'âge, le sexe et la région, a été recruté à partir d'un échantillon aléatoire obtenu de la base de données de la Régie de l'assurance maladie du Québec (RAMQ) des régions de Montréal, Laval et Sherbrooke (Québec, Canada). Les hommes et les femmes vivant dans la communauté et âgés de 67 à 84 ans ont été inclus dans l'étude s'ils parlaient français ou anglais, étaient disposés à s'engager pour une période de 5 ans, sans incapacité dans les activités de la vie quotidienne, sans atteinte cognitive (3MS>79; Canadian Study of Health and Aging Working Group, 1994) et capables de marcher 100 mètres et de monter 10 marches sans s'arrêter. Les personnes souffrant de maladies dégénératives à court terme (insuffisance cardiaque de classe II, maladie pulmonaire obstructive chronique exigeant une oxygénothérapie à domicile ou des stéroïdes oraux, cancer traité par radiothérapie, chimiothérapie ou chirurgie au cours des 5 dernières années) ou de maladies ayant un impact direct sur l'alimentation (maladie digestive inflammatoire) ont été exclues de l'étude (Gaudreau et al., 2007). De plus, à l'entrée dans l'étude, des données supplémentaires sur l'état dentaire ont été recueillies par quatre examinateurs ( 1 dentiste et 3 étudiantes en médecine dentaire) chez 113 participants, dont 94 ont passé un examen clinique d'efficacité masticatoire. Ce sous-échantillon de convenance a été recruté parmi les participants vivant à Sherbrooke afin de faciliter la collecte des données; ils proviennent de tous les quartiers de la ville.

\section{Collecte de données}

Les données ont été recueillies dans le cadre d'entrevues face-à-face réalisées par des infirmières et des diététistes spécialement formées. Un questionnaire comprenant les questions relatives à la capacité masticatoire tirées du Oral Health Impact Profile (OHIP, Slade \& Spencer, 1994) a été utilisé pour mesurer la perception masticatoire des participants $(n=1793)$.
L'efficacité masticatoire perçue a donc été déterminée à l'aide de 7 questions ayant chacune une échelle de Likert à 5 niveaux (toujours, souvent, occasionnellement, rarement, jamais) pour un score total de 28 points. Voici deux exemples de questions: «Durant les 4 dernières semaines, avez-vous eu de la difficulté à mastiquer des aliments à cause de problèmes avec vos dents, votre bouche ou vos prothèses?», «Durant les 4 dernières semaines, avez-vous été obligé d'interrompre un repas à cause de problèmes avec vos dents, votre bouche ou vos prothèses?». La mesure de l'efficacité masticatoire a été réalisée chez les participants du sous-échantillon ( $\mathrm{n}=94)$ à l'aide d'un test clinique qui évalue la performance d'une personne à mastiquer une carotte crue [Swallowing Threshold Test Index (STTI), score 0 à 100\%]. Le STTI développé par Chauncey, Muench, Kapur, et Wayler (1984) a été souvent utilisé (Demers et al., 1996; Mishellany-Dutour, Renaud, Peyron, Rimek, \& Woda, 2008; Shatenstein, 1986; Wayler \& Chauncey, 1983). La procédure est la suivante: le participant mastique $3 \mathrm{~g}$ de carotte crue pendant 30 cycles masticatoires sans consigne en ce qui a trait au côté de la bouche ni à la durée. Lorsqu'il est prêt à avaler, il le crache dans un tamis de grandeur prédéterminée. Cette procédure est répétée 3 fois et la bouche est rincée entre chaque essai pour récupérer toutes les particules mastiquées. Celles-ci sont par la suite centrifugées et le poids des particules alimentaires qui sont passées à travers le tamis est comparé au poids total de la nourriture mastiquée. Ce ratio, exprimé en $\%$, est employé comme mesure de performance masticatoire. Un score élevé indique une bonne efficacité masticatoire (Chauncey et al., 1984). Dans le cadre d'une entrevue diététique, le rappel alimentaire de 24 heures $(\mathrm{R} 24 \mathrm{H})$ fournit une description détaillée de tous les aliments solides et liquides consommés au cours des dernières $24 \mathrm{~h}$. Les intervieweurs ont utilisé des mesures graduées ainsi que des photographies de portions standards afin d'améliorer l'estimation des portions. Les apports habituels en protéines totales $(\mathrm{g} / \mathrm{kg} / \mathrm{j})$, vitamine $C(\mathrm{mg} / \mathrm{j})$, folate (équivalent de folate alimentaire, $\mu \mathrm{g} / \mathrm{j}$ ) ainsi que la consommation de fruits/légumes (nombre de portions/j) ont été calculés à partir de $3 \mathrm{R} 24 \mathrm{H}$ non-consécutifs réalisés sur 2 jours de semaine et une journée de fin de semaine, dont le premier a été colligé lors de l'entrevue et les 2 autres par téléphone. Nous avons choisi ces nutriments puisque ceux-ci proviennent généralement d'aliments plus difficiles à mastiquer comme la viande ou les noix (protéines) ou les fruits et les légumes crus (vitamine $\mathrm{C}$, folate). L'analyse nutritionnelle a été réalisée avec le logiciel d'analyse nutritionnel CANDAT (CANDAT, version 10, Godin London Inc), à partir du fichier canadien sur les éléments nutritifs (version 2007b), Santé Canada, enrichi d'environ 300 aliments par l'équipe de recherche de NuAge. 
L'utilisation de la moyenne de trois jours de rappels non consécutifs permet d'atténuer la variabilité intraindividuelle normalement observée dans la consommation alimentaire des individus et est une bonne estimation de l'alimentation habituelle (Payette \& GrayDonald, 1991). Les habitudes alimentaires ont été mesurées à l'aide de questions provenant de l'étude Santé Québec (Levasseur, 1987) portant sur la présence de changements, et sur les raisons de ces changements, dans les habitudes alimentaires, dans la quantité de nourriture consommée et dans les groupes d'aliments consommés. Le nombre total de conditions chroniques a été calculé en additionnant les conditions déclarées par les participants à l'aide du questionnaire Older American Resources and Services questionnaire (Fillenbaum \& Smyer, 1981) qui contient une liste de 17 maladies (score 0-17). L'autonomie dans les activités de la vie quotidienne et domestique, la mobilité, la communication et les fonctions mentales a été mesurée à l'aide du Système de mesure de l'autonomie fonctionnelle (SMAF, Hébert et al., 2003). Cet instrument détermine le niveau d'autonomie du participant selon un score total qui s'échelonne de 0 à 87; plus le score est élevé, plus la personne est dépendante. Les variables potentiellement associées à la fois à la santé dentaire et à la nutrition, telles que l'âge, le sexe, le nombre d'années de scolarité, le fait de vivre seul, la suffisance du revenu familial pour satisfaire aux besoins, le tabagisme, les conditions chroniques et l'autonomie fonctionnelle ont été prises en compte dans les analyses.

\section{Analyses statistiques}

Des analyses descriptives (moyenne \pm écart-type) ainsi que des pourcentages pour décrire la cohorte et le sous-échantillon ont été effectuées. Les différences entre les hommes et les femmes ont été testées par des tests $t$ ou chi carré selon la nature des variables. Les apports alimentaires et nutritionnels sont décrits selon les moyennes et les pourcentages d'apports insuffisants. Des analyses bivariées, pour examiner la relation entre les apports alimentaires et les variables de confusion (âge, sexe, vivre seul, scolarité, revenu, tabagisme, conditions chroniques et autonomie fonctionnelle), ont été exécutées tant pour la cohorte entière que pour le souséchantillon. Les variables significativement associées $(\mathrm{p}<0,05)$ ont été retenues pour les analyses subséquentes. Des analyses de régression linéaire multivariées ont été utilisées pour évaluer la relation entre l'efficacité masticatoire (mesurée et perçue) et les apports alimentaires en tenant compte des variables de confusion significativement associées avec la variable dépendante. Ces analyses ont été réalisées à l'aide du logiciel SAS 9.2 et toutes les données ont été pondérées pour l'âge, le sexe et la région selon le cas.

\section{Considérations éthiques}

Le protocole de recherche de cette étude secondaire a été approuvé par le comité d'éthique du CSSS-IUGS. Tous les participants de l'étude NuAge ont signé un formulaire de consentement après avoir été informés des objectifs de l'étude, des procédures et de leur droit de se retirer à tout moment du projet de recherche. Les formulaires de consentement soulignaient le fait que toutes les données seraient conservées dans une banque de données et que d'autres chercheurs seraient autorisés à réaliser des projets de recherche dans le domaine de la nutrition et du vieillissement. Toutes les données ont été dénominalisées et le fichier transféré selon un protocole établi par le comité directeur de NuAge.

\section{Résultats}

Les données sociodémographiques et de santé générale des participants du sous-échantillon et de la cohorte entière sont présentées, selon le sexe, dans le Tableau 1. Les participants de la cohorte NuAge étaient généralement instruits, avaient un revenu familial suffisant pour leurs besoins et étaient autonomes à leur entrée dans l'étude. Toutefois, plus de $75 \%$ ont déclaré avoir plus que trois conditions chroniques. Comparativement à la cohorte entière de NuAge, le souséchantillon $(n=94)$ était moins instruit $(10,8 \pm 0,4$ vs $13,0 \pm 0,2$ années de scolarité; $\mathrm{p}<0,001)$ et leur efficacité masticatoire perçue était plus faible $(24,7 \pm 0,5$ vs $26,0 \pm 0,2 ; p=0,008)$. Dans le sous-échantillon, les femmes étaient plus nombreuses que les hommes à avoir $\geq 3$ conditions chroniques et à vivre seules, et cela tant dans la cohorte entière que dans le souséchantillon. Par contre, elles avaient un nombre plus faible d'années de scolarité, étaient plus nombreuses à être non-fumeuses et étaient plus autonomes que les hommes seulement dans la cohorte totale. Le score moyen au test masticatoire était similaire chez les hommes (H) $(60,8 \%$ [IC $(57,3-64,2)])$ et les femmes (F) $(61,2 \%$ [IC $(57,7-64,7)])$. Dans ce sous-échantillon, $40 \%$ des participants ont une mauvaise efficacité masticatoire mesurée (score $<60 \%$ ), $31 \%$ se classe dans la catégorie acceptable (score $60-79,9 \%$ ) et $29 \%$ dans la catégorie excellente (score $\geq 80 \%$ ) (Chauncey et al., 1984). En revanche, les scores moyens d'efficacité masticatoire perçue sont élevés tant chez les participants du sous-échantillon $(\mathrm{H}: 25,26 \pm 0,63 / 28$; F: $24,31 \pm 0,65 / 28$ ) que de la cohorte entière de NuAge (H: 26,22 $\pm 0,19 / 28 ; \mathrm{F}: 25,81 \pm 0,21 / 28$ ). La description des apports alimentaires des participants est présentée dans les Tableaux 2 et 3 . Dans le sous-échantillon, comme dans la cohorte entière, la prévalence élevée d'apports alimentaires insuffisants en fruits et en légumes (recommandations de Santé Canada, 2008, 2010) se traduit par des niveaux d'apports insuffisants en folate 
Tableau 1 : Données sociodémographiques et de l'état de santé général des participants en fonction du sexe

\begin{tabular}{|c|c|c|c|c|c|c|}
\hline & \multicolumn{3}{|l|}{ Sous-échantillon } & \multicolumn{3}{|c|}{ Cohorte entière (sans le sous-échantillon) } \\
\hline Vit seul (\% oui) & 15,0 & 44,8 & $0,0026^{*}$ & 22,4 & 49,5 & $<0,0001^{2}$ \\
\hline $\begin{array}{l}\text { Nombre d'années de scolarité } \\
\text { Revenu satisfait les besoins (\%) }\end{array}$ & $11,6 \pm 0,8$ & $10,7 \pm 0,5$ & 0,3737 & $13,5 \pm 0,3$ & $12,7 \pm 0,2$ & $0,0192^{1}$ \\
\hline Pas très bien & 13,1 & 1,8 & & 5,9 & 8,4 & \\
\hline Pas du tout & - & - & & 0,7 & 0,3 & \\
\hline \multicolumn{7}{|l|}{ Tabagisme (\%) } \\
\hline Non-fumeur & 51,5 & 69,5 & 0,0951 & 33,0 & 68,2 & $<0,0001^{2}$ \\
\hline Ex-fumeur & 2,4 & 5,2 & & 11,0 & 5,0 & \\
\hline Fumeur & 46,1 & 25,2 & & 56,0 & 26,7 & \\
\hline$\geq 3$ & & & & 76,5 & 81,8 & \\
\hline Score SMAF ${ }^{3}$ & $4,8 \pm 0,7$ & $3,5 \pm 0,3$ & 0,1034 & $3,9 \pm 0,2$ & $3,2 \pm 0,1$ & $0,0041^{1}$ \\
\hline
\end{tabular}

1 test t.

2 test chi-deux.

3 Système de mesure de l'autonomie fonctionnelle, Score de $\mathbf{0}$ à 87 plus le score est élevé, plus la personne est dépendante.

* La mise en caractère gras indique les résultats significatifs.

chez les femmes. Des différences significatives existent entre les participants du sous-échantillon et de la cohorte entière en ce qui concerne les apports recommandés en fruits/légumes (sous-échantillon: 88,4\%; cohorte entière: $69,4 \% ; \mathrm{p}=0,0002$ ). Dans le sous-échantillon, tout comme dans la cohorte entière, on observe une différence entre les hommes et les femmes concernant l'apport moyen de folate (souséchantillon: $p=0,0310$; cohorte entière: $p<0,0001$ ). Des différences significatives sont également présentes entre les hommes et les femmes concernant l'apport recommandé en vitamine $C(p<0,0001)$ et folate $(\mathrm{p}<0,0001)$ dans la cohorte entière puis dans le sous-échantillon pour le folate $(p=0,0054)$. Par contre, aucune différence significative n'a été observée en ce qui a trait à l'apport protéinique. Dans le souséchantillon, le niveau de scolarité est négativement associé aux apports en vitamine $C(p=0,0125)$ et à la consommation de fruits/légumes ( $p=0,0147)$, alors que les apports en folate sont plus faibles chez les

Tableau 2 : Description des apports alimentaires et nutritionnels chez les participants du sous-échantillon en fonction du sexe

\begin{tabular}{|c|c|c|c|c|c|c|c|}
\hline $\begin{array}{l}\text { Apport alimentaire } \\
\text { moyen }\end{array}$ & $\begin{array}{l}\text { Hommes } \\
(n=41)\end{array}$ & $\begin{array}{l}\text { Femmes } \\
(n=53)\end{array}$ & test $t p$ & $\begin{array}{l}\text { Apport alimentaire } \\
\text { insuffisant }{ }^{1}\end{array}$ & $\begin{array}{l}\text { Hommes } \\
(n=41) \%\end{array}$ & $\begin{array}{l}\text { Femmes } \\
(n=53) \%\end{array}$ & $\chi^{2} \mathbf{p}$ \\
\hline $\begin{array}{l}\text { Protéines } \\
(\mathrm{g} / \mathrm{kg} / \mathrm{i})\end{array}$ & $1,1 \pm 0,05$ & $1,0 \pm 0,05$ & 0,2712 & $\begin{array}{l}\text { Protéines } \\
(H / F:<0,8 \mathrm{~g} / \mathrm{kg} / \mathrm{i})\end{array}$ & 19,5 & 22,7 & 0,7084 \\
\hline $\begin{array}{l}\text { Vitamine C } \\
(\mathrm{mg} / \mathrm{i})\end{array}$ & $100,1 \pm 8,1$ & $94,7 \pm 6,5$ & 0,6066 & $\begin{array}{l}\text { Vitamine } \mathbf{C}^{2} \\
(\mathrm{H}:<75 \mathrm{mg} / \mathrm{i} ; \mathrm{F}:<60 \mathrm{mg} / \mathrm{i})\end{array}$ & 32,0 & 22,3 & 0,2915 \\
\hline $\begin{array}{l}\text { Folate }^{2} \\
(\mu \mathrm{g} / \mathrm{i})\end{array}$ & $402,3 \pm 19,4$ & $347,2 \pm 15,8$ & 0,0310 * & $\begin{array}{l}\text { Folatate }^{2-3} \\
\qquad(\mathrm{H} / \mathrm{F}:<320 \mu \mathrm{g} / \mathrm{i})\end{array}$ & 22,1 & 50,2 & 0,0054 \\
\hline $\begin{array}{l}\text { Fruits et légumes } \\
\text { (nombre de portions/i) }\end{array}$ & $5,0 \pm 0,4$ & $4,6 \pm 0,3$ & 0,4292 & $\begin{array}{l}\text { Fruits et légumes } \\
(H / F:<7 \text { portions/i) }\end{array}$ & 87,5 & 89,0 & 0,8248 \\
\hline
\end{tabular}

1 Santé Canada. (2008). Aliments et nutrition-Combien de portions du Guide alimentaire de Légumes et fruits dois-je consommer?. Récupéré le 30 avril 2014 sur le site de l'organisme : http://www.hc-sc.gc.ca/fn-an/food-guide-aliment/choose-choix/fruit/ need-besoin-fra.php; Santé Canada. (2010). Aliments et nutrition- Apports nutritionnels de référence. Récupéré le 30 avril 2014 du site de l'organisme : http://www.hc-sc.gc.ca/fn-an/nutrition/reference/table/index-fra.php.

2 Besoins moyens estimés (Santé Canada, 2010).

3 Équivalent de folate alimentaire.

* La mise en caractère gras indique les résultats significatifs. 
Tableau 3 : Description des apports alimentaires et nutritionnels chez les participants de la cohorte entière (sans le sous-échantillon) en fonction du sexe

\begin{tabular}{|c|c|c|c|c|c|c|c|}
\hline $\begin{array}{l}\text { Apport alimentaire } \\
\text { moyen }\end{array}$ & $\begin{array}{l}\text { Hommes } \\
(n=812)\end{array}$ & $\begin{array}{l}\text { Femmes } \\
(n=887)\end{array}$ & test t p & $\begin{array}{l}\text { Apport alimentaire } \\
\text { insuffisant }^{1}\end{array}$ & $\begin{array}{l}\text { Hommes } \\
(n=812) \%\end{array}$ & $\begin{array}{l}\text { Femmes } \\
(\mathbf{n}=887) \%\end{array}$ & $\chi^{2} \mathbf{p}$ \\
\hline $\begin{array}{l}\text { Protéines } \\
\text { (g/kg/i) }\end{array}$ & $1,1 \pm 0,02$ & $1,1 \pm 0,02$ & 0,7880 & $\begin{array}{l}\text { Protéines } \\
\qquad(H / F:<0,8 \mathrm{~g} / \mathrm{kg} / \mathrm{i})\end{array}$ & 20,2 & 21,3 & 0,7084 \\
\hline $\begin{array}{l}\text { Vitamine C } \\
(\mathrm{mg} / \mathrm{i})\end{array}$ & $125,8 \pm 4,4$ & $133.9 \pm 3.8$ & 0,1623 & $\begin{array}{l}\text { Vitamine } \mathbf{C}^{2} \\
\quad(\mathrm{H}:<75 \mathrm{mg} / i ; \mathrm{F}:<60 \mathrm{mg} / \mathrm{i})\end{array}$ & 25,1 & 14,1 & $<0,0001$ \\
\hline $\begin{array}{r}\text { Folate }^{2} \\
(\mu \mathrm{g} / \mathrm{i})\end{array}$ & $432,0 \pm 9,2$ & $383,3 \pm 6,7$ & $<0,0001$ * & $\begin{array}{l}\text { Folate }^{2-3} \\
\qquad(\mathrm{H} / \mathrm{F}:<320 \mu \mathrm{g} / \mathrm{i})\end{array}$ & 21,7 & 57,5 & $<0,0001$ \\
\hline
\end{tabular}

1 Santé Canada. (2008). Aliments et nutrition-Combien de portions du Guide alimentaire de Légumes et fruits dois-je consommer?. Récupéré le 30 avril 2014 sur le site de l'organisme : http://www.hc-sc.gc.ca/fn-an/food-guide-aliment/choose-choix/fruit/ need-besoin-fra.php ${ }_{i}$ Santé Canada. (2010). Aliments et nutrition- Apports nutritionnels de référence. Récupéré le 30 avril 2014 du site de l'organisme : h\#p://www.hc-sc.gc.ca/fn-an/nutrition/reference/table/index-fra.php.

2 Besoins moyens estimés (Santé Canada, 2010).

3 Équivalent de folate alimentaire.

* La mise en caractère gras indique les résultats significatifs.

femmes ( $p=0,031)$ ainsi que chez les personnes moins autonomes $(p=0,0363)$. Dans la cohorte entière, l'autonomie fonctionnelle est négativement associée aux apports en protéines $(\mathrm{p}<0,0001)$ et aux apports en vitamine $C(p=0,0036)$. Puis le niveau de scolarité est positivement associé aux apports en vitamine $C$ $(\mathrm{p}=0,0076)$. Des apports significativement plus élevés en folate sont observés chez les hommes ( $p<0,0001)$ ainsi que les personnes moins âgées $(\mathrm{p}<0,0001)$ ayant moins de conditions chroniques $(p=0,0378)$, étant plus autonomes $(\mathrm{p}=0,0009)$ et plus scolarisées $(p=0,0189)$. Les personnes vivant seules $(p=0,0109)$, étant plus autonomes $(\mathrm{p}=0,0363)$ et plus scolarisées $(p=0,0005)$ ont des apports en fruits/légumes plus élevés. Le score d'efficacité masticatoire mesurée n'est pas significativement associé aux apports alimentaires, même après ajustement pour l'âge, le sexe, le niveau de scolarité et l'autonomie fonctionnelle (Tableau 4). De même, l'efficacité masticatoire perçue n'est pas significativement associée aux apports alimentaires, même après ajustement pour l'âge, le sexe, le fait de vivre seul, la scolarité, l'autonomie fonctionnelle et les conditions chroniques (Tableau 5). De plus, les variables d'efficacité masticatoire perçue et mesurée ne sont pas significativement associées aux apports alimentaires insuffisants, même après ajustement pour les variables de confusion. En ce qui concerne les habitudes alimentaires, on observe qu'une forte proportion des participants (45\%) déclare ne manger que rarement ou jamais certains groupes d'aliments, comme les poissons, les aliments très riches en gras, les fruits de mer, les aliments acides et les fruits/légumes (Tableau 6). Parmi eux, un seul participant invoque la difficulté à mastiquer ou à avaler, et 7 autres mentionnent la difficulté à digérer, comme raison pour exclure ces aliments. Vingt-huit pour cent des participants déclarent que certaines conditions influencent leurs habitudes alimentaires mais un seul identifie un problème dentaire en lien avec ces comportements.

\section{Discussion}

Dans le cadre de cette analyse réalisée auprès d'un large échantillon de la population âgée vivant dans la communauté, aucune relation significative n'a été observée entre l'efficacité masticatoire, qu'elle soit mesurée ou perçue, et la consommation de fruits/légumes ou les apports de vitamine $C$, de folate et de protéines.

Tableau 4 : Relation entre les apports alimentaires et l'efficacité masticatoire mesurée ( $n=94)$

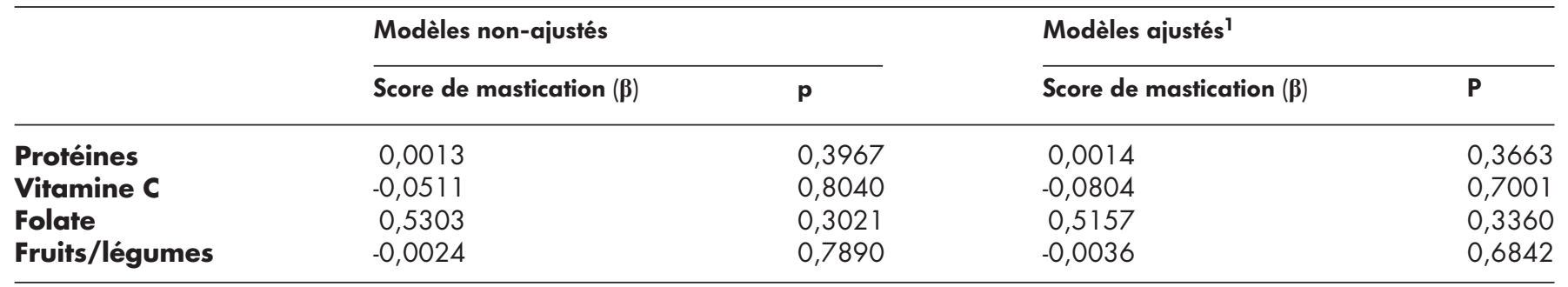

1 modèles ajustés selon l'âge, le sexe, le niveau de scolarité et l'autonomie fonctionnelle. 
Tableau 5 : Relation entre les apports alimentaires et l'efficacité masticatoire perçue

\begin{tabular}{|c|c|c|c|c|c|c|}
\hline & \multicolumn{3}{|c|}{ Modèles non-ajustés } & \multicolumn{3}{|c|}{ Modèles ajustés ${ }^{1}$} \\
\hline Vitamine C & 1682 & 1,2383 & 0,0735 & 1677 & 1,0931 & 0,1157 \\
\hline Folate & 1682 & 2,3692 & 0,1037 & 1566 & 1,4741 & 0,3124 \\
\hline Fruits/légumes & 1680 & 0,0312 & 0,3024 & 1675 & 0,0308 & 0,3309 \\
\hline
\end{tabular}

1 modèles ajustés selon l'âge, le sexe, le niveau de scolarité, le fait de vivre seul, l'autonomie fonctionnelle et les conditions chroniques.

Il est à noter que, même dans cette population généralement en bonne santé, la majorité des participants ne consomment pas la quantité recommandée de fruits et de légumes et cela se réflète sur une prévalence élevée d'apports insuffisants en folate chez les femmes. Finalement, alors que la performance au test clinique d'efficacité masticatoire est faible, la perception de cette efficacité dans la population étudiée est élevée. Il est possible que la mesure clinique et la perception de l'efficacité masticatoire rendent compte de deux réalités différentes. Dans une étude précédente, Cusson et al. (2015) ont mis en évidence une absence de relation entre la mesure directe de l'efficacité masticatoire et sa perception. En effet, les participants ne percevaient aucun problème masticatoire alors que le test montrait le contraire. Le phénomène d'adaptation pourrait expliquer ces résultats de même que ceux présentés dans cet article. En réalité, les participants ayant une efficacité masticatoire réduite pourraient avoir modifié leurs habitudes alimentaires en regard des choix d'aliments ou des modes de cuisson et ainsi maintenir leurs apports nutritionnels. Par exemple, une personne pourrait remplacer les viandes fibreuses par la viande hachée, les oeufs et les produits laitiers afin de combler ses besoins protéiniques. Bien que l'analyse des habitudes alimentaires montre que peu de gens ont modifié leurs habitudes en raison de problèmes masticatoires, près du tiers des participants rapporte avoir modifié leurs habitudes alimentaires pour d'autres raisons. Ces modifications d'habitudes alimentaires les ont peut-être conduits à manger des aliments plus mous, plus faciles à mastiquer faisant en sorte qu'ils ne ressentent pas de problèmes masticatoires.

De plus, il n'y a pas d'association entre le résultat au test clinique et la qualité des apports alimentaires. En effet, les personnes ayant un résultat élevé au test clinique n'ont pas d'apports plus élevés que celles ayant une moindre performance au test. Le test clinique utilisé nous donne des mesures objectives, toutefois il ne reflète peut-être pas la perception masticatoire des participants et il ne semble pas lié aux apports alimentaires examinés dans cette étude (Cusson et al., 2015). Ce test mesure la capacité de l'appareil masticateur à broyer la nourriture. La performance masticatoire est jugée selon la proportion de particules mastiquées qui ont atteint le critère déterminé (particules plus petites que $4 \mathrm{~mm}$ ). Toutefois, à notre connaissance, ce critère n'a pas été testé en fonction de l'impact de la grosseur des particules mastiquées sur le confort lors de la mastication ou de la déglutition ni sur l'alimentation et la digestion des personnes âgées. Quelques auteurs ont montré que l'état dentaire n'influençait pas l'état nutritionnel tel que mesuré par l'apport de nutriments, l'anthropométrie ou la biochimie, mais plutôt le choix des aliments. Par exemple, Ervin et Dye (2009) n'ont trouvé aucune association significative entre l'état dentaire et l'apport de nutriments, notamment la vitamine $C$ et le carotène. Nordenram, Ryd-Kjellen, Johansson, Nordstrom, et Winblad (1996) ont montré que l'état nutritionnel, estimé à partir de l'anthropométrie et l'albumine sérique, ne semblait pas influencé par l'état dentaire,

Tableau 6 : Analyse descriptive des habitudes alimentaires chez les participants du sous-échantillon (n=94)

\begin{tabular}{|c|c|c|c|c|}
\hline $\begin{array}{l}\text { Y-a-t-il quelque chose qui } \\
\text { influence vos habitudes } \\
\text { alimentaires? }\end{array}$ & oui: $n=26$ & $\begin{array}{l}\text { régime thérapeutique: } n=7 \\
\text { maladie: } n=7 \\
\text { problèmes digestifs: } n=3 \\
\text { prothèse dentaire: } n=1\end{array}$ & & \\
\hline
\end{tabular}


mais que le choix des aliments, notamment d'après leur consistance, était relié à l'état dentaire. En effet, avoir ses dents naturelles et des PDO est important dans le choix de la nourriture (Nordenram et al., 1996). Liedberg, Stoltze, Norlén, et Öwall (2007) n'ont observé aucune différence significative entre les participants ayant une diète adéquate ou inadéquate quant au nombre de dents et de contacts occlusaux, à la présence de prothèses dentaires ou à la mastication perçue (Liedberg et al., 2007). Par contre, de faibles apports en fruits et légumes ont été observés chez les sujets ayant une faible performance masticatoire (Brodeur et al., 1993; Lee et al., 2010) et chez les sujets édentés (Marcenes et al., 2003). Dans la présente étude, nous avons considéré des nutriments ainsi que des aliments (fruits / légumes). Malgré cela, nous n'avons pas observé de relation entre l'apport en fruits/légumes et l'efficacité masticatoire, qu'elle soit perçue ou mesurée. Tel que mentionné précédemment, ceci pourrait être dû à des changements d'habitudes alimentaires (type de cuisson, exclusion d'aliments et leur remplacement par des aliments plus faciles à mastiquer) et à l'adaptation des participants à leur condition dentaire.

\section{Forces et limites}

La cohorte NuAge a été recrutée à partir d'un échantillon aléatoire et stratifiée pour l'âge, le sexe et la région. Par contre, le sous-échantillon en est un de convenance. Dans ce cas, un biais de sélection pourrait être présent, même si des mesures ont été prises pour que tous les quartiers de la ville de Sherbrooke, donc tous les niveaux socio-économiques, soient représentés. Toutefois, les participants du sous-échantillon étaient moins instruits et la perception de leur efficacité masticatoire était plus faible comparativement à la cohorte NuAge entière. Un échantillon plus grand et représentatif aurait peut-être permis de détecter des associations significatives. La qualité du protocole pour la collecte de données assure des mesures précises et valides. La méthode des trois R24h non-consécutifs permet une estimation valide des apports alimentaires habituels chez les personnes âgées (Payette et al., 1999). Le test masticatoire utilisé est administré selon un protocole rigoureux. Comme les résultats de cette étude transversale n'ont pas permis d'observer de relation entre l'efficacité masticatoire, et les apports alimentaires et nutritionnels, une étude longitudinale pourrait permettre d'observer les effets délétères à plus long terme d'une piètre efficacité masticatoire sur l'alimentation des personnes âgées.

\section{Conclusion}

Les résultats de cette étude fournissent des pistes permettant d'améliorer la méthodologie de recherche en santé dentaire en lien avec la santé nutritionnelle. Les futures études devraient ainsi tenir compte de la consistance des aliments consommés et considérer d'autres aliments-clés essentiels à une diète équilibrée et habituellement plus difficiles à mastiquer tel que la viande et les noix. Une meilleure connaissance de l'impact à long terme de la santé dentaire sur l'état nutritionnel des personnes âgées reste à acquérir.

\section{Références}

Arpin, S., Brodeur, J.-M., \& Corbeil, P. (2008). Dental caries, problems perceived and use of services among institutionalized elderly in 3 regions of Quebec, Canada. Journal of the Canadian Dental Association, 74(9), 807-807d.

Brodeur, J.-M., Laurin, D., Vallée, R., \& Lachapelle, D. (1993). Nutrient intake and gastrointestinal disorders related to masticatory performance in the edentulous elderly. Journal of Prosthetic Dentistry, 70(5), 468-473.

Canadian Study of Health and Aging Working Group. (1994). The Canadian study of health and aging: Study methods and prevalence of dementia. Canadian Medical Association Journal, 150, 899-913.

Centre d'excellence pour la santé buccodentaire et le vieillissement. Université Laval Faculté de médecine dentaire. (2006). Mandat et mécanismes de travail. p. 15.

Chauncey, H. H., Muench, M. E., Kapur, K. K., \& Wayler, A. H. (1984). The effect of the loss of teeth on diet and nutrition. International Dental Journal, 34, 98-104.

Cusson, V., Caron, C., Gaudreau, P., Morais, J. A., Shatenstein, B., \& Payette, H. (2015). Assessing older adults' masticatory efficiency. Journal of the American Geriatrics Society, 63(6), 1192-1196.

Demers, M., Bourdages, J., Brodeur, J.-M., \& Benigeri, M. (1996). Indicators of masticatory performance among elderly complete denture wearers. The Journal of Prosthetic Dentistry, 75(2), 188-193.

Ervin, R. B., \& Dye, B. A. (2009). The effect of functional dentition on healthy eating index scores and nutrient intakes in a nationally representative sample of older adults. Journal of Public Health Dentistry, 69(4), 207-216.

Fédération Dentaire Internationale. (1982). Global goals for oral health in the year 2000. International Dental Journal, 32(1), 74-77.

Fillenbaum, G. G., \& Smyer, M. A. (1981). The development, validity and reliability of the OARS multidimensional functional assessment questionnaire. The Journals of Gerontology, 36, 428-434.

Gaudreau, P., Morais, J. A., Shatenstein, B., Gray-Donald, K., Khalil, A., Dionne, I., et al. (2007). Nutrition as a determinant of successful aging: Description of the Quebec longitudinal study NuAge and results from cross-sectional pilot studies. Rejuvenation Research, 10(3), 377-386.

Gonsalves, W. C., Wrightson, A. S., \& Henry, R. G. (2008). Common oral conditions in older persons. American Family Physician, 78(7), 845-852. 
Hébert, R., Desrosiers, J., Dubuc, N., Tousignant, M., Guilbeault, J., \& Pinsonnault, E. (2003). Le Système de Mesure de l'Autonomie Fonctionnelle (SMAF). La Revue de Gériatrie, 28(4), 323-336.

Keller, H., Østbye, T., \& Goy, R. (2004). Nutritional risk predicts quality of life in elderly community-living canadians. Journal of Gerontology, 59A(1), 68-74.

Lee, M.-S., Huang, Y.-C., \& Wahlquist, M. L. (2010). Chewing ability in conjunction with food intake and energy status in later life affects survival in Taiwanese with the metabolic syndrome. Journal of the American Geriatrics Society, 58(6), 1072-1080.

Levasseur, M. (1987). Sources et justifications des questions utilisées dans l'Enquête Santé Québec. Cahier technique 87-03. Ministère de la Santé et des Services sociaux, Gouvernement du Québec: http:/ / www.stat.gouv.qc.ca/ publications / sante/sante 1.htm.

Liedberg, B., Stoltze, K., Norlén, P., \& Öwall, B. (2007). Inadequate dietary habits and mastication in elderly men. Gerodontology, 24, 41-46.

Marcenes, W., Steele, J. G., Sheiham, A., \& Walls, A. W. G. (2003). The relationship between dental status, food selection, nutrient intake, nutritional status, and body mass index older people. Cadernos de Saúde Pública, 19(3), 809-816.

Mishellany-Dutour, A., Renaud, J., Peyron, M.-A., Rimek, F., \& Woda, A. (2008). Is the goal of mastication reached in young dentates, aged dentates and aged denture wearers? British Journal of Nutrition, 99, 121-128.

N'Gom, P. I., \& Woda, A. (2002). Influence of impaired mastication on nutrition. The Journal of Prosthetic Dentistry, $87,667-673$

Nordenram, G., Ryd-Kjellen, E., Johansson, G., Nordstrom, G., \& Winblad, B. (1996). Alzheimer's disease, oral function and nutritional status. Gerodontology, 13(1), 9-16.

Ordre des dentistes du Québec. (2007). Consultation publique sur les conditions de vie des aînés. Montréal, Canada: Ordre des dentistes du Québec.

Payette, H., \& Gray-Donald, K. (1991). Dietary intake and biochemical indices of nutritional status in an elderly population with estimates of the precision of the 7-d food record. The American Journal of Clinical Nutrition, 54, $478-488$.

Ramage-Morin, P. L., \& Garriguet, D. (2013). Risque nutritionnel chez les Canadiens âgés - Travaux de recherche. Statistique Canada, Catalogue no 82-003-X, Rapports sur la santé, 24(3), 3-14.

Rose, L. F., Steinberg, B. J., \& Minsk, L. (2000). The relationship between periodontal disease and systemic conditions.
Compendium of continuing education in dentistry, 21(10A), 870-877.

Sahyoun, N. R., Lin, C.-L., \& Krall, E. (2003). Nutritional status of the older adult is associated with dentition status. Journal of the American Dietetic Association, 103(1), 61-66.

Santé Canada. (2008). Aliments et nutrition-Combien de portions du Guide alimentaire de Légumes et fruits dois-je consommer? Récupéré le 30 avril 2014 sur le site de l'organisme : http:/ / www.hc-sc.gc.ca/fn-an/food-guide-aliment/choosechoix/fruit/need-besoin-fra.php

Santé Canada. (2009). Effets de la santé buccodentaire sur l'état de santé général. Récupéré le 12 février 2011 du site de l'organisme : http://www.hc-sc.gc.ca/hl-vs/iyh-vsv/ life-vie/dent-fra.php

Santé Canada. (2010). Aliments et nutrition-Apports nutritionnels de référence. Récupéré le 30 avril 2014 du site de l'organisme: http:/ /www.hc-sc.gc.ca/fn-an/nutrition/ reference/table/index-fra.php

Shatenstein, B. (1986). Tooth loss, mastication, and nutrition: An overview. Journal of the American Dietetic Association, 86(1), 86-87.

Shay, K. (2002). Infectious complications of dental and periodontal diseases in the elderly population. Clinical Infectious Diseases, 34, 1215-1223.

Sheiham, A., Steele, J. G., Marcenes, W., Finch, S., \& Walls, A. W. G. (1999). The impact of oral health on stated ability to eat certain foods; findings from the national diet and nutrition survey of older people in Great Britain. Gerodontology, 16(1), 11-20.

Slade, G. D., \& Spencer, A. J. (1994). Development and evaluation of the oral health impact profile. Community Dental Health, 11(1), 3-11.

Canada, Statistique. (2011). Canadiens en contexte - Vieillissement de la population. Récupéré le 12 février 2011 du site de l'organisme : http://www4.hrsdc.gc.ca/.3ndic.1t.4r@fra.jsp?iid=33

Taylor, G. W., Loesche, W. J., \& Terpenning, M. S. (2000). Impact of oral diseases on systemic health in the elderly: Diabetes mellitus and aspiration pneumonia. Journal of Public Health Dentistry, 60(4), 313-320.

Wayler, A. H., \& Chauncey, H. H. (1983). Impact of complete dentures and impaired natural dentition on masticatory performance and food choice in healthy aging men. The Journal of Prosthetic Dentistry, 49(3), 427-433.

Yoshihara, A., Watanabe, R., Nishimuta, M., Hanada, N., \& Miyazaki, H. (2005). The relationship between dietary intake and the number of teeth in elderly Japanese subjects. Gerodontology, 22, 211-218. 